\title{
Video Article \\ Cell Based Assays of SINEUP Non-coding RNAs That Can Specifically Enhance mRNA Translation
}

\author{
Hazuki Takahashi* ${ }^{1}$, Harshita Sharma* ${ }^{1}$, Piero Carninci ${ }^{1,2}$ \\ ${ }^{1}$ Laboratory for Transcriptome Technology, RIKEN Center for Integrative Medical Sciences \\ ${ }^{2}$ TransSINE Technologies Co. Ltd. \\ *These authors contributed equally
}

Correspondence to: Piero Carninci at carninci@riken.jp

URL: https://www.jove.com/video/58627

DOI: doi:10.3791/58627

Keywords: Genetics, Issue 144, SINEUPs, antisense RNA, long noncoding RNAs (IncRNAs), translation regulation, high-throughput screening, RNA therapy, antibody production

Date Published: 2/1/2019

Citation: Takahashi, H., Sharma, H., Carninci, P. Cell Based Assays of SINEUP Non-coding RNAs That Can Specifically Enhance mRNA Translation. J. Vis. Exp. (144), e58627, doi:10.3791/58627 (2019).

\section{Abstract}

Targeted-protein enhancement is of importance not only for the study of biological processes but also for therapeutic and biotechnological applications. Here, we present a method to selectively up-regulate protein expression of desired genes in cultured cells by means of synthetic antisense non-coding RNAs known as SINEUPs. This positive control of gene expression is at the post-transcriptional level and exerted by an inverted short interspersed nuclear element (SINE) repeat at the 3' end of SINEUPs that comprises its effector domain (ED). SINEUPs can specifically bind to any protein-coding mRNA of choice through its binding domain (BD), a region designed to complement the sequence within the $5^{\prime}$ untranslated region (5' UTR) and around the start codon of the mRNA. Target-specific SINEUPs designed in this manner are transfected to cultured cells, and protein and RNA are extracted for downstream analyses, generally $24-48 \mathrm{~h}$ post-transfection. SINEUP-induced protein upregulation is detected by Western-blot analysis and RNA expression is measured using real-time quantitative reverse transcription PCR. We have observed that BD design is critical for achieving optimum SINEUP activity and that testing different BD sizes and positions with respect to the start codon of the target mRNA is recommended. Therefore, we describe here a semi-automated high-throughput imaging method based on fluorescence detection that can be implemented to target mRNA fused with green fluorescent protein (GFP). SINEUPs specifically enhance translation within normal physiological range of the cell, without altering the target transcript level. This method has been successfully employed against a range of endogenous and exogenous targets, in a wide variety of human, mouse, and insect cell lines along with in vivo systems. Moreover, SINEUPs have been reported to increase antibody production and work as an RNA therapeutic against haploinsufficient genes. The versatile and modular nature of SINEUPs makes them a suitable tool for gene-specific translational control.

\section{Video Link}

The video component of this article can be found at https://www.jove.com/video/58627/

\section{Introduction}

In the post-genomic era, many insights have been gained into the gene regulatory roles of non-coding antisense transcripts owing to the development of next-generation sequencing technologies ${ }^{1,2,3}$ and gene-editing tools. This category of transcripts, which was previously considered to be "transcriptional trash", is now established as a key player of genetic regulation. Antisense transcripts are reported to modulate chromatin and control stability and expression of their cognate protein-coding sense $\mathrm{mRNA}^{4,5}$. In most cases, this mode of regulation is negative and antisense transcripts silence their sense counterparts through RNA-RNA interactions ${ }^{6,7}$. This trait of natural antisense transcripts has been utilized to downregulate desired genes in the form of synthetic small interfering RNA (siRNA), microRNA (miRNA), and antisense oligos $(\mathrm{ASO})^{8,9,10,11}$ leaving a technological void for targeted gene up-regulation.

One intriguing study changed the perspective of the antisense RNA field by demonstrating that antisense long non-coding RNAs (AS IncRNAs) of the genes Uchl1 (ubiquitin C-terminal hydrolase L1) and Uxt (ubiquitously-expressed transcript) positively regulate translation of their cognate sense mRNA at the post-transcriptional level in mice ${ }^{12}$. The $5^{\prime}$ end of these AS IncRNAs overlaps with several bases in the $5^{\prime}$ untranslated region (5' UTR) of their corresponding sense transcripts, and the non-overlapping 3 ' end contains an inverted repeat of a retrotransposon belonging to the short interspersed nuclear element (SINE) family. Interestingly, we found that the main driving force behind this translational up-regulation is the embedded SINE repeat and it is not limited to mouse SINE repeats only. Human Alu repeat-containing AS IncRNAs also enhanced translation of target sense mRNAs, reinforcing the idea of a novel class of SINE-driven regulatory antisense non-coding RNAs, appropriately named SINEUPs ${ }^{13}$. Recent studies showed that the potential of natural SINEUPs is preserved in synthetic SINEUPs designed to specifically target various endogenous and exogenous genes ${ }^{14,15}$. SINEUPs have two important features: first the "binding domain" (BD) which is generally the $5^{\prime}$ end region complementary to the sequence encompassing the primary start codon of a protein-coding mRNA, and second the "effector domain" (ED) as it incorporates an inverted repeat of SINE which is a prerequisite for SINEUP function ${ }^{14}$ (Figure 1). SINEUPS can be customized to design a BD specifically targeting a gene of choice. This can then be harnessed to dissect the function of a particular 
gene involved in a biological pathway, as a better alternative to a conventional mRNA overexpression strategy. In addition, this versatile tool can be applied to boost antibody production, and as a drug to treat haploinsufficient diseases caused by insufficient dosages of functional proteins $^{16,17,18,19,20,21}$.

The advantages of SINEUP technology are manifold. It does not alter the expression of the target at the transcriptional level. Longer BDs provide more specificity to SINEUPs, while not inducing a dsRNA-dependent stress response ${ }^{15}$. The protein induction is maintained within the normal physiological range of the cell, preventing any deleterious effect due to aberrant or excessive protein expression. SINEUPs are compatible with a wide variety of mouse, human, and hamster cultured cell lines, for instance, HEK293T/17, HepG2, HeLa, CHO, MN9D, and many more ${ }^{12,13,14,15,18,19}$. SINEUPs can efficiently target endogenous genes, transiently overexpressed genes, and FLAG-tagged or luciferasefusion genes, eliminating the need of gene-specific antibodies ${ }^{14,18}$. The basic SINEUP analysis requires routine cell culture, transfection, sodium dodecyl sulfate-polyacrylamide gel electrophoresis (SDS-PAGE), real time quantitative reverse transcription-polymerase chain reaction (qRT$\mathrm{PCR}$ ) instruments and settings, and expertise can be gained in a short time.

Here, we describe a method for targeting genes with synthetic SINEUPs to up-regulate translation, an effect contrary to other technologies such as RNA interference ${ }^{9}$ and ASO gapmers ${ }^{11,22,23}$. A widely-used method for RNA-guided gene activation is clustered regularly interspaced short palindromic repeats-based activation (CRISPRa), where the gene expression is triggered at the transcriptional level ${ }^{24}$. This method, though simple and fast, requires multiple single guide RNAs (sgRNAs) targeting the same gene for higher efficiency, thereby increasing the chances of off-target binding ${ }^{25}$. In addition, the key enzyme of the CRISPRa system, the catalytically dead CRISPR-associated protein 9 (dCas9) has low binding specificity and sgRNAs targeting bi-directional promoter regions can non-specifically up-regulate nearby genes ${ }^{25}$. Conversely, SINEUPs bind to target mRNA at a single binding region with high specificity and do not affect expression of nearby genes. We discuss the basic steps involved in SINEUP design, transfection, protein and RNA expression analyses. Furthermore, we present a semi-automated, high-throughput imaging system to screen multiple SINEUPs at once, which is useful for detection of optimum SINEUPs.

\section{Design of BDs of SINEUP Constructs for Target mRNAs}

1. Check transcription starting site (TSS) and translation starting site of target mRNAs in the cells of interest. Acquire TSS data from both the ENCODE and FANTOM projects (cap analysis of gene expression, CAGE) (ZENBU: http://fantom.gsc.riken.jp/zenbu/).

2. Open the browser, search genes of interest and check TSS by CAGE peaks.

3. Consult the example analysis of cell and tissue specific TSS of Parkinson disease protein 7 (PARK7) mRNA shown in Figure 2.

4. Design BD sequences of several different lengths corresponding to 40 bases upstream and 32 bases downstream of the first methionine (AUG), $72 \mathrm{nt}$ in total.

5. Custom order the designed BDs in SINEUP expression vector (see Table of Materials). NOTE: Check the sequence specificity by basic local alignment search tool (BLAST) to avoid off-target effects ${ }^{26}$.

\section{Cell Culture and SINEUP Transfection}

1. Seed cells of interest in a 6 well-plate or 24 well-plate for $24 \mathrm{~h}$ before transfection of SINEUPs. In the case of human embryonic kidney cell line (HEK293T/17) (see Table of Materials), seed $0.5 \times 10^{6}$ cells per well of a 6 well-plate or $1.5 \times 10^{5}$ cells per well of a poly-D-lysine coated 24 well-plate (see Table of Materials). It becomes $70 \%$ confluent after $24 \mathrm{~h}$.

2. After $24 \mathrm{~h}$, change the medium to $1.5 \mathrm{~mL}$ of fresh medium for a 6 well-plate or $0.4 \mathrm{~mL}$ of fresh medium for a 24 well-plate. In case of SINEUPGFP, transfect $0.6 \mu \mathrm{g}$ of pEGFP-C2 (see Table of Materials) and $3.4 \mu \mathrm{g}$ of SINEUP-GFP in one well of a 6 well-plate or $130 \mathrm{ng}$ of pEGFPC2 and $670 \mathrm{ng}$ of SINEUP-GFP in one well of a 24 well-plate with transfection reagent (10 $\mu \mathrm{L}$ in 6 well-plate and $3 \mu \mathrm{L}$ in 24 well-plate) by following the manufacturer's instructions (see Table of Materials), and incubate at $37^{\circ} \mathrm{C}$ in a $5 \% \mathrm{CO} 2$ incubator for $24 \mathrm{~h}$.

3. Wash cells with $2 \mathrm{~mL}$ of phosphate buffer saline (PBS) $\left(37 \mathrm{mM} \mathrm{NaCl}, 8 \mathrm{mM} \mathrm{Na} 2 \mathrm{HPO} 4,2.6 \mathrm{mM} \mathrm{KCl}\right.$ and $1.5 \mathrm{mM} \mathrm{KH}_{2} \mathrm{PO}$ ) in each well of a 6 well-plate or $0.5 \mathrm{~mL}$ of PBS in each well of a 24 well-plate. Only for poly-D-lysine coated 24 well-plate, add $25 \mu \mathrm{L}$ of $0.05 \%$ weight per volume Trypsin, incubate in a $5 \% \mathrm{CO}_{2}$ incubator for $5 \mathrm{~min}$ and add $275 \mu \mathrm{L}$ of cell medium per well. In case of 6 well-plate, add $2 \mathrm{~mL}$ of PBS in each well. Pipet up and down to harvest $3 / 4$ of cells $(1.5 \mathrm{~mL}$ for a 6 well-plate or $225 \mu \mathrm{L}$ for a 24 well-plate) from 1 well for protein extraction (go to protocol 3 for protein extraction) and $1 / 4$ of cells $(0.5 \mathrm{~mL}$ for a 6 well-plate or $75 \mu \mathrm{L}$ for a 24 well-plate) for RNA extraction and centrifuge at $6,000 \times \mathrm{g}$ for $5 \mathrm{~min}$ at $4^{\circ} \mathrm{C}$ (go to step 5 for RNA extraction).

NOTE: Analyze the effect of SINEUP-GFP in a poly-D-lysine coated 24 well-plate by imaging. Go to step 7 (imaging analysis).

\section{Protein Extraction}

1. Carefully aspirate $1.5 \mathrm{~mL}$ of PBS and add $140 \mu \mathrm{L}$ of lysis solution $\left(20 \mathrm{mM}\right.$ Tris- $\mathrm{HCl}(\mathrm{pH} 7.5), 150 \mathrm{mM} \mathrm{NaCl}, 1 \mathrm{mM} \mathrm{Na}{ }_{2} \mathrm{EDTA}, 1 \mathrm{mM}$ EGTA, $1 \%$ (w/v) Triton, $2.5 \mathrm{mM}$ sodium pyrophosphate, $1 \mathrm{mM} \beta$-glycerophosphate, $1 \mathrm{mM} \mathrm{Na} \mathrm{VO}_{4}, 1 \mu \mathrm{g} / \mathrm{mL}$ leupeptin, and $0.005 \%$ (w/v) phenylmethylsulfonyl fluoride (PMSF)) (see Table of Materials) for a 6 well-plate or $60 \mu \mathrm{L}$ for a 24 well-plate to the cell pellets.

2. Mix by pipetting and then mix thoroughly by rotating at slow speed for $1 \mathrm{~h}$ at $4{ }^{\circ} \mathrm{C}$. Collect supernatant by centrifugation at $14,000 \times g$ for 10 $\min$ at $4{ }^{\circ} \mathrm{C}$

3. Check protein concentration by colorimetric assay (see Table of Materials). Prepare all the reactions at room temperature.

1. Prepare 5-6 times dilution of bovine serum albumin (BSA) protein standard in ultrapure water with concentrations ranging from 0.2-1.5 $\mathrm{mg} / \mathrm{mL}$ protein. Prepare fresh standards each time.

2. Prepare working reagent $A^{\prime}$ by mixing $1 \mathrm{~mL}$ of reagent $A$ (alkaline copper tartrate solution) with $20 \mu \mathrm{L}$ of reagent $S$ (surfactant solution).

3. Load $5 \mu \mathrm{L}$ of water (negative control), BSA standard (protein standard and positive control) or protein sample in each well of a $96-$ wellplate. 
4. Add $25 \mu \mathrm{L}$ of reagent $\mathrm{A}^{\prime}$ in each well. Carefully add $200 \mu \mathrm{L}$ of reagent $\mathrm{B}$ (Folin reagent) per well avoiding any bubble formation. Cover the plate with aluminum foil and wait for 5-10 min.

5. Measure the protein absorbance at $750 \mathrm{~nm}$ with a spectrophotometer. The absorbance is stable for at least $1 \mathrm{~h}$.

6. Prepare standard curve by plotting BSA standard protein concentrations $(\mathrm{mg} / \mathrm{mL})$ on the $\mathrm{x}$-axis and their respective absorbance on the $y$-axis. Calculate sample protein concentration by applying standard curve equation.

NOTE: Pause the protocol at this step if needed or go to step 4. For long-term storage, snap freeze protein samples in liquid nitrogen and store at $-80^{\circ} \mathrm{C}$

\section{Protein Separation by SDS-PAGE and Detection of Target Proteins with Antibodies (Western-blot Analysis)}

1. Add one volume of $2 x$ loading dye $(0.1 \mathrm{M}$ Tris- $\mathrm{HCl}(\mathrm{pH} 6.8), 4 \%$ SDS, $20 \%$ glycerol, $1.42 \% 2$-mercaptethanol and $0.2 \%$ bromophenol blue) to each volume of protein sample from step 3.3. Heat at $90{ }^{\circ} \mathrm{C}$ for $5 \mathrm{~min}$ and immediately cool on ice for $1 \mathrm{~min}$.

2. Load $10-20 \mu \mathrm{g}$ of protein samples to $10 \%$ SDS poly-acrylamide gel and separate at $100-150 \mathrm{~V}$ ) (see Table of Materials)

3. Transfer protein from gel to $0.45-\mu \mathrm{m}$ nitrocellulose membrane (see Table of Materials) by semi-dry transfer instrument with transfer buffer (25 mM Tris, $192 \mathrm{mM}$ glycine and $20 \%$ methanol) at $25 \mathrm{~V}$ for $30 \mathrm{~min}$.

4. Add blocking solution ( $5 \%$ non-fat dry milk in $1 \times$ TBST buffer (137 mM NaCl, $20 \mathrm{mM} \mathrm{Tris-HCl} \mathrm{(pH} \mathrm{7.6),} \mathrm{0.1 \%} \mathrm{Tween-20))} \mathrm{(see} \mathrm{Table} \mathrm{of}$ Materials) to the container until the membrane is completely soaked and incubate it at room temperature for 30 min.

5. In the case of SINEUP-GFP, hybridize the protein with anti-GFP antibody (diluted 1:2000 in blocking solution) (see Table of Materials) by incubating the membrane for 30 min with shaking at room temperature. As an internal control protein, detect $\beta$-actin protein by hybridizing with Anti- $\beta$-Actin antibody (diluted 1:2000 in blocking solution) (see Table of Materials) for 30 min with shaking at room temperature.

6. Add $1 x$ TBST buffer to the container until the membrane is completely soaked and wash for 5 min at room temperature. Repeat the wash step two more times (a total of three washes).

7. Hybridize GFP protein to diluted (1:1000 in blocking solution) anti-rabbit antibody conjugated with horseradish peroxidase (HRP), and hybridize $\beta$-actin protein to diluted (1:1000 in blocking solution) anti-mouse antibody conjugated with HRP on the membrane for 30 min with shaking at room temperature.

8. Wash membrane with $1 \times$ TBST buffer for $5 \mathrm{~min}$ at room temperature. Repeat the wash step two more times (a total of three washes).

9. Mix equal volume of HRP-enhanced chemiluminescence (ECL) detection reagent 1 and 2 (see Table of Materials). Transfer the membrane to $2 \mathrm{~mL}$ ECL reagent mix, cover the box with aluminum foil and let it incubate for 1-2 min at room temperature. Carefully remove the membrane from ECL reagent mix and expose using a luminescence imaging instrument.

\section{RNA Extraction and DNase Treatment}

1. Extract total RNA following the standard protocol for RNA extraction from cells grown in a monolayer ${ }^{27}$ or alternatively use a commercially available RNA extraction kit (see Table of Materials). To state in brief, add any monophasic lysis reagent (MLR) of guanidine isothiocyanate and phenol to the harvested cells in step 2.3 ( $1 \mathrm{~mL}$ MLR per $\sim 5$ million cells $)^{27}$.

NOTE: Change gloves frequently. Use RNase-free reagents, and diethylpyrocarbonate (DEPC)-treated plastic ware and glassware to prevent RNA degradation. Pause the protocol at this step if needed. Store extracted RNA at $-80^{\circ} \mathrm{C}$.

2. Add 0.1 volume of $10 x$ DNase buffer and $2 U$ of DNase to the purified RNA from step 5.1 (see Table of Materials).

3. Set the reaction volume to $50 \mu \mathrm{L}$ with nuclease-free water and mix gently. Incubate at $37^{\circ} \mathrm{C}$ for $30 \mathrm{~min}$.

4. Add 0.1 volume of re-suspended DNase inactivation reagent and mix well (see Table of Materials). Incubate for 5 min at room temperature with gentle shaking.

5. Centrifuge at $10,000 \times g$ for $90 \mathrm{~s}$. Transfer the supernatant (DNA-free RNA) to a fresh RNase-free $1.5 \mathrm{~mL}$ tube. Be careful not to touch the pellet because this can cause carry-over of DNase which is troublesome for downstream steps.

6. Quantitate RNA by measuring $\mathrm{A}_{260}$ and $\mathrm{A}_{260} / \mathrm{A}_{280}$ ratio (for pure RNA, the range is 1.8-2.0) in a UV spectrophotometer.

\section{First Strand cDNA Synthesis and qRT-PCR Analysis}

1. Perform first strand cDNA synthesis following a standard cDNA synthesis protocol as below (see Table of Materials).

1. Mix $0.75 \mu \mathrm{M}$ oligo dT primer, $4.3 \mu \mathrm{M}$ random primer, dNTPs (10 mM each) with 200-500 ng total RNA (from steps $5-8$ ) in $10 \mu \mathrm{L}$ of total reaction volume. Incubate at $65^{\circ} \mathrm{C}$ for $5 \mathrm{~min}$ and then immediately put on ice.

2. Mix $1 \times$ reverse transcriptase buffer, $1 \cup$ of RNase inhibitor, $10 \cup$ of reverse transcriptase, and add to $10 \mu \mathrm{L}$ RNA-primer mix from 6.1.1.

3. Set the total reaction volume to $20 \mu \mathrm{L}$ with nuclease-free water if required. Mix gently and incubate the reaction mix at $30{ }^{\circ} \mathrm{C}$ for $10 \mathrm{~min}$, then at $42{ }^{\circ} \mathrm{C}$ for $60 \mathrm{~min}$, and finally at $95^{\circ} \mathrm{C}$ for $5 \mathrm{~min}$ to inactivate the enzyme. Cool the tubes on ice.

NOTE: Thaw RNA samples and all the reagents on ice and prepare the reaction mix on ice. If target RNAs are only polyA RNAs, use oligo dT primer only $(2.5 \mu \mathrm{M})$. Pause the protocol if needed. Store synthesized cDNA at $-20^{\circ} \mathrm{C}$

2. Perform qRT-PCR in technical triplicates $\left(n=3, C_{T}\right.$ standard deviation $\left.>0.2\right)$ and within biological replicates $(n \geq 3)$ using $1 \mu L$ of 10 ng cDNA, $0.4 \mu \mathrm{L}$ of reverse primer $(10 \mu \mathrm{M}), 0.4 \mu \mathrm{L}$ of forward primer $(10 \mu \mathrm{M}), 10 \mu \mathrm{L}$ of mixture solution of DNA polymerase, $0.4 \mu \mathrm{L}$ of double-strand DNA staining dye, $10 \mu \mathrm{L}$ of nuclease-free water (reaction volume was $20 \mu \mathrm{L}$ ) to detect PCR products using following conditions; hold stage $(1 \mathrm{cycle})$ for $30 \mathrm{~s}$ at $95^{\circ} \mathrm{C}$, cycling stage $\left(40\right.$ cycles) for $5 \mathrm{~s}$ at $95^{\circ} \mathrm{C}$ and for $30 \mathrm{~s}$ at $60^{\circ} \mathrm{C}$, melt curve stage. Primer examples are for Human Gapdh Fw: TCTCTGCTCCTCCTGTTC, Human Gapdh Rv: GCCCAATACGACCAAATCC, EGFP Fw: GCCCGACAACCACTACCTGAG, EGFP Rv: CGGCGGTCACGAACTCCAG, SINEUP-GFP Fw: CTGGTGTGTATTATCTCTTATG and SINEUP-GFP_Rv: CTCCCGAGTCTCTGTAGC. See Table of Materials. Analyze quantitative RNA expression with $2^{-\Delta \Lambda C t} \pm S^{2}$ method ${ }^{28}$. 


\section{Imaging Analysis of SINEUPs by Semi-automated Detection Method}

1. Wash cells with $0.5 \mathrm{~mL}$ of PBS for one well of a 24 well-plate

2. Add $2.0 \mu \mathrm{g}$ of Hoechst 33342 (see Table of Materials) (dissolving in $500 \mu \mathrm{L}$ of PBS) to each well of the 24-well-plate and incubate cells at 37 ${ }^{\circ} \mathrm{C}$ for 20 min to stain the nucleus.

3. Measure Hoechst stained cells to count the total number of cells at a blue emission maximum of $461 \mathrm{~nm}$, and intensity of green fluorescence to count number of GFP positive cells at a green emission maximum of $510 \mathrm{~nm}$ by using a high-throughput micro-well image cytometer (see Table of Materials). Analyze protein up-regulatory effect of the SINEUPs by calculating GFP integrated intensity, which is the sum of all pixel intensities displaying signal in segmented objects calculated for each channel, using Imaging software ${ }^{29}$.

4. Harvest cells to check RNA expression (back to steps 5 and 6 ).

\section{Representative Results}

SINEUP-GFP is a synthetic SINEUP containing both an optimum BD (-28/+4 overlap to GFP mRNA) and an ED (inverted SINE B2 from AS-Uch/1) that can up-regulate GFP mRNA translation (Figure 3A and 3B) without changing the expression of GFP mRNA (Figure 3C and 3D). To screen optimum BDs and EDs for SINEUPs, we developed a protocol of semi-automated image analysis that improved detection time and increased the number of samples being simultaneously screened compared with a conventional Western-blot analysis ${ }^{15}$. As shown in Figure 4, this high-throughput imaging system took 3 days (Western-blot analysis took 2 weeks for 48 SINEUPs). Having demonstrated that SINEUPGFP increases GFP translation, we detected GFP integrated intensity by the image cytometer. The optimum BD of SINEUP-GFP induced a 1.4fold increase in GFP protein expression. Although we observed compression of signals from 2.6-fold (Western-blot analysis, see Figure 3A) to 1.4-fold (Imaging analysis, see Figure 5), the difference might be due to the calibration of the imaging instrument software. Nevertheless, we detected significantly higher levels of GFP fluorescence compared with the control (Figure 5A and 5B).

\section{Target mRNA}

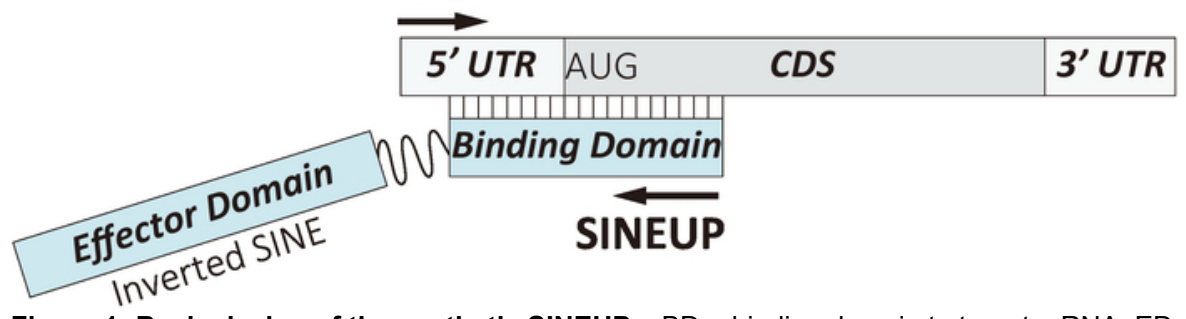

Figure 1: Basic design of the synthetic SINEUPs. BD = binding domain to target mRNA, ED = effector domain containing an inverted SINE sequence. Please click here to view a larger version of this figure. 
A

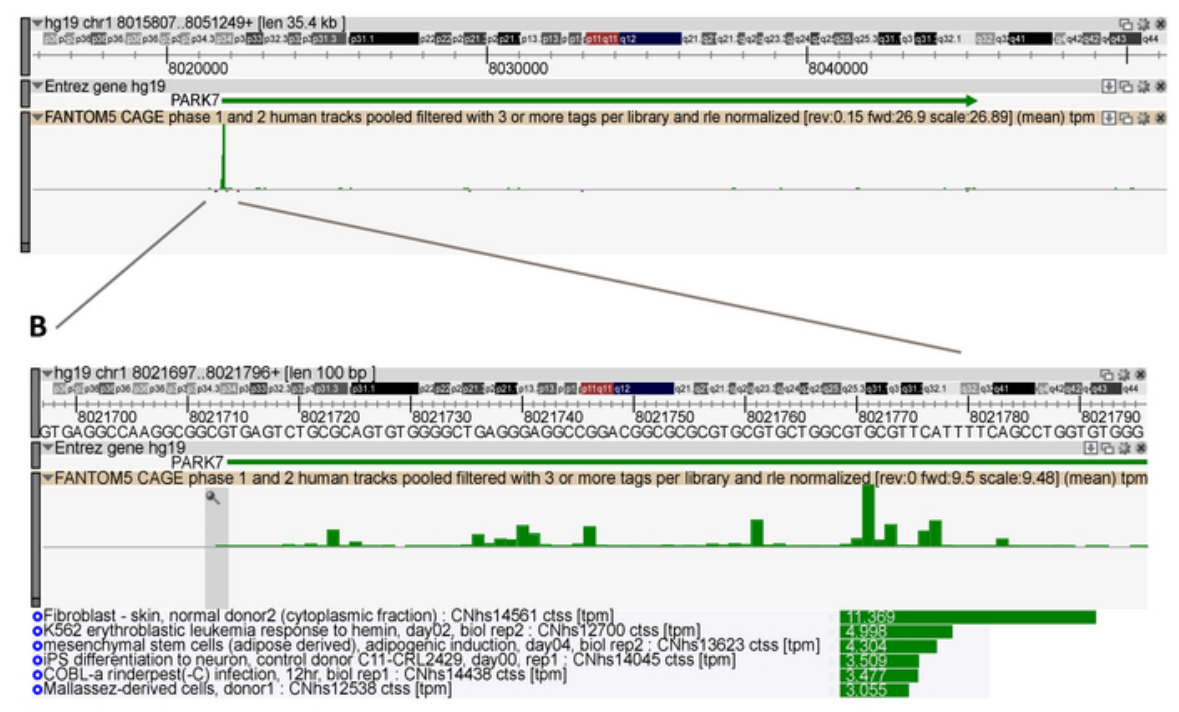

Figure 2: Transcription starting sites (TSSs) of human Parkinson disease protein 7 (PARK7) mRNA in specific tissues and cells as detected by CAGE analysis. (A) A snapshot showing a TSS search result for human PARK7 mRNA using an omics data integration and interactive visualization system ${ }^{30}$. The horizontal green arrow in the Entrez gene hg19 track indicates the genomic position of the reference human PARK7 mRNA and the vertical green bars in the FANTOM5 CAGE 1 and 2 tracks indicate the sum of TSSs (measured as transcripts per million (tpm)) from 1,829 types of tissues and cells. (B) Zoom in of the marked region of the TSS in panel A (1/354 scale: $35.4 \mathrm{~kb}$ to $100 \mathrm{bp}$ ). Grey shaded area in the FANTOM5 CAGE phase 1 and 2 tracks indicates the TSS of 6 specific cells out of the total 1,829, which are listed at the bottom of the figure. The numbers $(11.369,4.998,4.304,3.509,3.477$, and 3.055$)$ corresponding to these listed cells indicate transcripts per million for each specific cell at the grey shaded positions of the TSS. Please click here to view a larger version of this figure. 
A

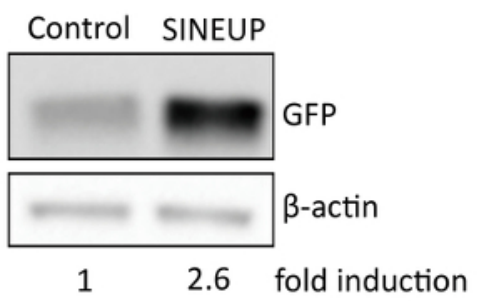

B

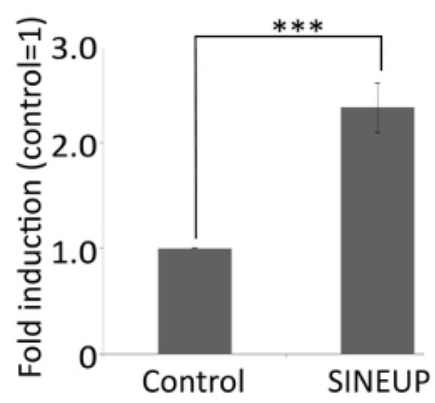

C GFP mRNA

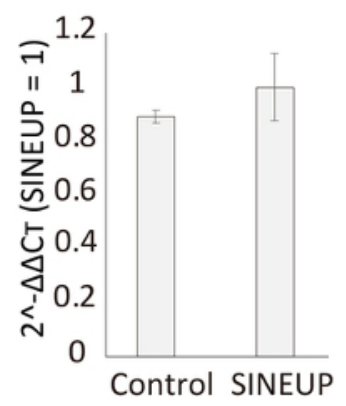

D

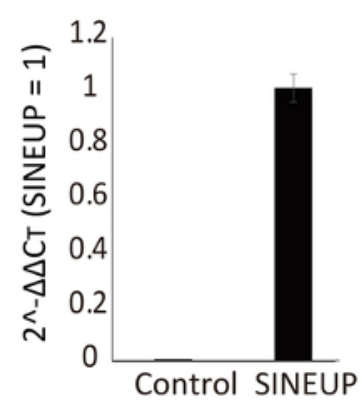

Figure 3: Western-blot analysis confirming up-regulation of translation by SINEUP-GFP. (A) SINEUP-GFP was examined by Westernblot with anti-GFP antibody. (B) Results were normalized to the intensity of the $\beta$-actin protein band. (C) GFP mRNA and (D) SINEUP RNAs expression were detected by qRT-PCR. ${ }^{* * *} p<0.0005, n=3$, two-tailed student's t-test, error bars are standard deviation. This figure has been modified from Takahashi et al. ${ }^{15}$. Please click here to view a larger version of this figure.

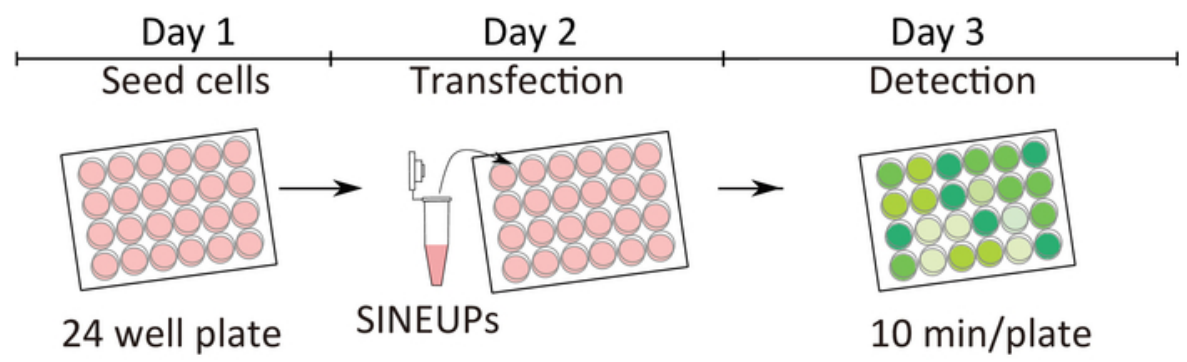

Figure 4: Schematic of SINEUP semi-automated image analysis. Day 1: Seed cells of interest in a 24 well-plate. Day 2: Transfect GFP and SINEUP-GFP. Day 3: Analyze the effect of SINEUPs by measuring GFP integrated intensity. Please click here to view a larger version of this figure. 
A

Control

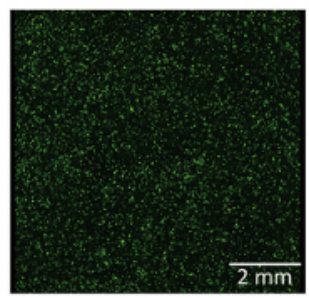

SINEUP

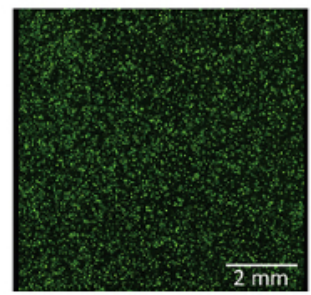

\section{4-well plate 1 well FOV 16}

B

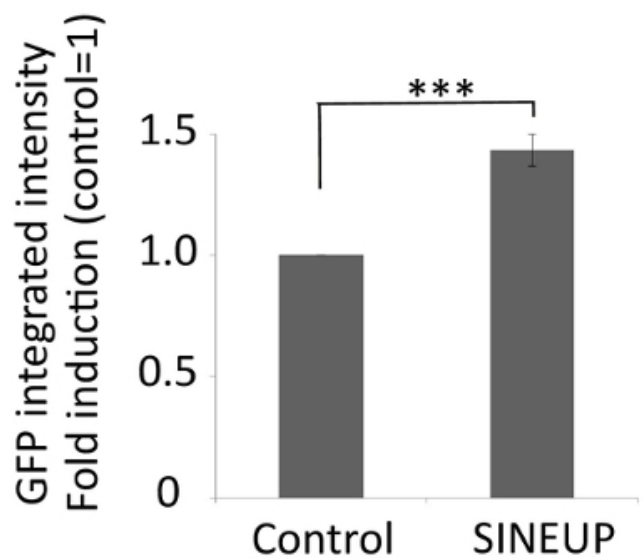

Figure 5: High-throughput analysis of translation up-regulation by SINEUP-GFP. (A) Comparison of GFP fluorescence between control and SINEUP-GFP transfected cells ${ }^{15}$. Scale bar $=2 \mathrm{~mm}$. (B) GFP integrated intensity was normalized to the total cell number counted by nuclear staining with Hoechst 33342 . ${ }^{* *} \mathrm{p}<0.0005, \mathrm{n}=3$, two-tailed student's t-test, error bars are standard deviation. FOV: field of view. This figure has been modified from Takahashi et al. ${ }^{15}$. Please click here to view a larger version of this figure.

\section{Discussion}

We described here a protocol to specifically enhance protein production of a target mRNA by means of a SINE-containing non-coding RNA, called SINEUPs. As a representative example, optimum synthetic SINEUP-GFP is shown to up-regulate the translation of GFP mRNA 2.6-fold as measured by Western-blot analysis.

Designing an optimum BD is crucial to ensure SINEUP specificity and potency (extent of protein up-regulation). Previously, we screened 17 BDs of SINEUP-GFP by Western-blot analysis ${ }^{15}$ and found that the optimum BD overlaps the AUG-KOZAK sequence of GFP mRNA, though it may not be the case with other mRNAs and should be verified for each case. Another independent group also screened the BD using a different method $^{31}$. As screening many BDs can be quite time-consuming and cumbersome, we introduced a high-throughput SINEUP detection method here. This method measures relative changes in GFP integrated density in SINEUP transfected-cells compared to the control vector transfectedcells. To ensure that cells in a particular well of a culture plate are transfected equally and GFP signal is not concentrated to only a certain region of the well, it is very important to distribute the cells equally in the wells in step 2.1. For this purpose, gently shake the plate 10 times back and forth $(\uparrow \downarrow)$ after seeding the cells inside a clean bench and repeat in the $5 \% \mathrm{CO}_{2}$ incubator before starting the incubation.

Another critical step is the calculation of protein concentration in step 3.3. Miscalculations here can lead to the loading of an erroneous amount of protein during Western-blot analysis, consequently preventing detection of small changes in protein expression by some of the weak SINEUPs or generating false positives from overloading. It is recommended to freshly prepare the protein standard curve every time, making sure that equal amounts of standards and protein samples are measured in step 3.3.3. The protocol described here focuses on SINEUP-GFP, but Western-blot analysis can be used for any target mRNA of interest. The incubation time and concentration of antibodies should be optimized for each target to get the best result.

One of the unique features of SINEUPs is that target-mRNA expression level remains unaffected. It is important to treat RNA with DNase to avoid detection of transfected SINEUPs and genomic DNA by qRT-PCR. SINEUP RNAs and target mRNA expression should be measured by qRT-PCR to confirm the success of both transfection and SINEUP activity. SINEUPs contain a SINE sequence, which is abundant throughout 
both the human and mouse genomes. To avoid non-specific detection of SINE sequences, it is not recommended to design qRT-PCR primers to the SINE sequence.

In this protocol we used human cell lines, but SINEUPs are efficacious in a number of cell lines from several different species ${ }^{12,13,14,18,19}$. The cell culture and transfection conditions can be modified according to different cell lines as long as these maintain transfection efficiency of the SINEUP vectors. Moreover, alternative methods of RNA extraction, cDNA synthesis, and protein concentration checking can be employed, given that they preserve the required RNA and protein quality for qRT-PCR and Western-blot analysis. While we used a specific high-throughput micro-well cytometer, which enabled detection of GFP fluorescence across the entire well, other cytometers with a similar detection range can be used $^{31}$. It is to be noted that if the distribution of cells and transfection are equal throughout a well, then it is not necessary to scan the whole well for GFP fluorescence: half or one-quarter of the area of a well might be enough to discern SINEUP effect depending on experimental skills.

Establishing a high-throughput SINEUP detection protocol allows for simultaneous screening of multiple BDs targeting a given mRNA in cultured cells. This is important as the rules governing optimum targeting by the BD are still unclear. Such a multiplex screening system allows for largescale testing of many SINEUPs against different genes, useful for targeting multiple genes involved in a particular signaling pathway for instance. Furthermore, it can be utilized to expand the search for effective SINEUPs targeting several mRNAs, designing different SINEUP BDs around AUG-Kozak region (see Figure 1), co-transfecting full length target mRNAs (5' UTR-CDS-3' UTR) fused with GFP mRNA in cultured cells to find the optimum SINEUPs, and subsequently testing BD candidates against endogenous mRNA in cultured cells and in vivo model animals, from humans and mice to other animal and plant species.

This screening protocol is very fast. We do not need to fix and collect cells, but just need to place the living cell culture plate in the imaging instrument. We propose to use this high-throughput screening protocol to select optimum BDs of SINEUPs and evaluate potential candidates by Western-blot analysis. Thus, selected candidates with optimum BDs can be applied to increase antibody production ${ }^{18,19,21}$. Currently, the RNA therapeutic field is dramatically growing. For instance, siRNA, ASO, mRNA, and CRISPR RNA therapies, are widely employed to control mRNA expression of their respective targets ${ }^{9,32}$. In this context, SINEUPs are in their infancy, but so far none of the studied SINEUPs changed expression of target mRNAs. In addition, SINEUPs do not edit target mRNA, but only up-regulate translation of mRNA. Furthermore, loss-of-function diseases resulting from haploinsufficiency can be targeted by SINEUP therapy, achieving a 2-fold induction of the deficient protein $^{17,33}$. Although off-target effects need to be further studied, SINEUPs potentially and specifically target a single, expressed mRNA with a complementary sequence to the BD.

A limitation of this high-throughput protocol is that it is not suitable to screen BDs of SINEUPs in in vivo mouse models because the protocol measures the GFP integrated intensity only. As SINEUPs are natural antisense IncRNAs that act post-transcriptionally, they cannot be applied when the target mRNA is missing in the cells or tissue samples.

Nevertheless, SINEUPs can be applied to gain-of-function studies, to enhance antibody production, and as an RNA therapy to up-regulate expression of deficient proteins within the range of 1.5-3.0-fold. The methods described here present a useful guide to target and detect SINEUP-induced translation enhancement of desired mRNA and provide a new tool for post-transcriptional gene regulation.

\section{Disclosures}

The corresponding author Piero Carninci is one of the founders of TransSINE Technologies Co. Ltd. that holds intellectual property on filed patent (EP2691522A4 and JP2017169573A), and granted patent (US9353370B2) for SINEUP technology.

\section{Acknowledgments}

This study was partly supported by the Basic Science and Platform Technology Program for Innovative Biological Medicine, no. 17am0301014, from the Japan Agency for Medical Research and Development (AMED), Research Grant from MEXT to the RIKEN Center for Life Science Technologies and a Research Grant from MEXT to the RIKEN IMS. We thank members of the PC lab and of the SINEUP network (SISSA, University of Eastern Piedmont and RIKEN) for thought-provoking discussions. We thank Dr. Matthew Valentine for proofreading.

\section{References}

1. Katayama, S. et al. Antisense transcription in the mammalian transcriptome. Science. 309 (5740), 1564-1566 (2005).

2. Carninci, P. et al. The transcriptional landscape of the mammalian genome. Science. 309 (5740), 1559-1563 (2005).

3. Hon, C.C. et al. An atlas of human long non-coding RNAs with accurate 5' ends. Nature. 543 (7644), 199-204 (2017).

4. Tufarelli, C. et al. Transcription of antisense RNA leading to gene silencing and methylation as a novel cause of human genetic disease. Nature Genetics. 34 (2), 157-165 (2003).

5. Yu, W. et al. Epigenetic silencing of tumour suppressor gene p15 by its antisense RNA. Nature. 451 (7175), 202-206 (2008).

6. Carninci, P., Hayashizaki, Y. Noncoding RNA transcription beyond annotated genes. Current Opinion in Genetics \& Development. 17 (2), 139-144 (2007).

7. Chu, Y., Yue, X., Younger, S.T., Janowski, B.A., Corey, D.R. Involvement of argonaute proteins in gene silencing and activation by RNAs complementary to a non-coding transcript at the progesterone receptor promoter. Nucleic Acids Research. 38 (21), 7736-7748 (2010).

8. Ha, M., Kim, V.N. Regulation of microRNA biogenesis. Nature Reviews Molecular Cell Biology. 15 (8), $509-524$ (2014).

9. Takahashi, H., Carninci, P. Widespread genome transcription: new possibilities for RNA therapies. Biochemical and Biophysical Research Communications. 452 (2), 294-301 (2014).

10. Lam, J.K.W., Chow, M.Y.T., Zhang, Y., Leung, S.W.S. siRNA Versus miRNA as Therapeutics for Gene Silencing. Molecular Therapy-Nucleic Acids. 4 e252 (2015).

11. Nishina, K. et al. DNA/RNA heteroduplex oligonucleotide for highly efficient gene silencing. Nature communications. 6 7969 (2015). 
12. Carrieri, C. et al. Long non-coding antisense RNA controls Uchl1 translation through an embedded SINEB2 repeat. Nature. 491 (7424), 454-457 (2012).

13. Schein, A., Zucchelli, S., Kauppinen, S., Gustincich, S., Carninci, P. Identification of antisense long noncoding RNAs that function as SINEUPs in human cells. Scientific Reports. 633605 (2016).

14. Zucchelli, S. et al. SINEUPs are modular antisense long non-coding RNAs that increase synthesis of target proteins in cells. Frontiers in Cellular Neuroscience. 9174 (2015).

15. Takahashi, H. et al. Identification of functional features of synthetic SINEUPs, antisense IncRNAs that specifically enhance protein translation. PLOS ONE. 13 (2), e0183229 (2018).

16. Deutschbauer, A.M. et al. Mechanisms of haploinsufficiency revealed by genome-wide profiling in yeast. Genetics. 169 (4), 1915-1925 (2005).

17. Indrieri, A. et al. Synthetic long non-coding RNAs [SINEUPs] rescue defective gene expression in vivo. Scientific Reports. 6 27315 (2016).

18. Patrucco, L. et al. Engineering mammalian cell factories with SINEUP noncoding RNAs to improve translation of secreted proteins. Gene. (2015).

19. Sasso, E. et al. A long non-coding SINEUP RNA boosts semi-stable production of fully human monoclonal antibodies in HEK293E cells. MAbs. 1-8 (2018).

20. Dang, V.T., Kassahn, K.S., Marcos, A.E., Ragan, M.A. Identification of human haploinsufficient genes and their genomic proximity to segmental duplications. European Journal of Human Genetics. 16 (11), 1350-1357 (2008).

21. Zucchelli, S., Patrucco, L., Persichetti, F., Gustincich, S., Cotella, D. Engineering Translation in Mammalian Cell Factories to Increase Protein Yield: The Unexpected Use of Long Non-Coding SINEUP RNAs. Computational and Struct Biotechnology Journal. 14, 404-410 (2016).

22. Hua, Y. et al. Antisense correction of SMN2 splicing in the CNS rescues necrosis in a type III SMA mouse model. Genes \& Development. 24 (15), 1634-1644 (2010).

23. Watts, J.K., Corey, D.R. Silencing disease genes in the laboratory and the clinic. Journal of Pathology. 226 (2), $365-379$ (2012).

24. Perez-Pinera, P. et al. RNA-guided gene activation by CRISPR-Cas9-based transcription factors. Nature Methods. 10 (10), $973-976$ (2013).

25. Wu, X. et al. Genome-wide binding of the CRISPR endonuclease Cas9 in mammalian cells. Nature Biotechnology. 32 (7), $670-676$ (2014).

26. Altschul, S.F., Gish, W., Miller, W., Myers, E.W., Lipman, D.J. Basic local alignment search tool. Journal of Molecular Biology. 215 (3), 403-410 (1990).

27. Liu, X., Harada, S. RNA isolation from mammalian samples. Current Protocols in Molecular Biology. Chapter 4 Unit 4.16 (2013).

28. Livak, K.J., Schmittgen, T.D. Analysis of relative gene expression data using real-time quantitative PCR and the 2(-Delta Delta C(T)) Method. Methods. 25 (4), 402-408 (2001).

29. Schneider, C.A., Rasband, W.S., Eliceiri, K.W. NIH Image to ImageJ: 25 years of image analysis. Nature Methods. 9 (7), $671-675$ (2012).

30. Severin, J. et al. Interactive visualization and analysis of large-scale sequencing datasets using ZENBU. Nature Biotechnology. 32 (3), 217-219 (2014).

31. Yao, Y. et al. RNAe: an effective method for targeted protein translation enhancement by artificial non-coding RNA with SINEB2 repeat. Nucleic Acids Research. 43 (9), e58 (2015).

32. Savić, N., Schwank, G. Advances in therapeutic CRISPR/Cas9 genome editing. Translational Research. 168 15-21 (2016).

33. Long, H. et al. RNAe in a transgenic growth hormone mouse model shows potential for use in gene therapy. Biotechnology Letters. (2016). 